\title{
A Bayesian Approach to Jointly Estimate Tire Radii and Vehicle Trajectory
}

\author{
Emre Özkan, Christian Lundquist and Fredrik Gustafsson
}

\section{Linköping University Post Print}

N.B.: When citing this work, cite the original article.

(C2010 IEEE. Personal use of this material is permitted. However, permission to reprint/republish this material for advertising or promotional purposes or for creating new collective works for resale or redistribution to servers or lists, or to reuse any copyrighted component of this work in other works must be obtained from the IEEE.

Emre Özkan, Christian Lundquist and Fredrik Gustafsson, A Bayesian Approach to Jointly Estimate Tire Radii and Vehicle Trajectory, 2011, Proceedings of the International IEEE Conference on Intelligent Transportation Systems, 1-6.

http://dx.doi.org/10.1109/ITSC.2011.6082980

Postprint available at: Linköping University Electronic Press

http://urn.kb.se/resolve?urn=urn:nbn:se:liu:diva-72977 


\title{
A Bayesian Approach to Jointly Estimate Tire Radii and Vehicle Trajectory
}

\author{
Emre Özkan, Christian Lundquist and Fredrik Gustafsson
}

\begin{abstract}
High-precision estimation of vehicle tire radii is considered, based on measurements on individual wheel speeds and absolute position from a global navigation satellite system (GNSS). The wheel speed measurements are subject to noise with time-varying covariance that depends mainly on the road surface. The novelty lies in a Bayesian approach to estimate online the time-varying radii and noise parameters using a marginalized particle filter, where no model approximations are needed such as in previously proposed algorithms based on the extended Kalman filter. Field tests show that the absolute radius can be estimated with millimeter accuracy, while the relative wheel radius on one axle is estimated with submillimeter accuracy.
\end{abstract}

\section{INTRODUCTION}

Tire pressure monitoring has become an integral part of todays' automotive active safety concept. With the announcement of US standard (FMVSS 138) and European standard (ECE R-64) vehicle manufacturer must provide a robust solution to early detect tire pressure loss. A direct way to measure the tire pressure is by equipping the wheel with a pressure sensor and transmitting the information wireless to the body. This is costly and therefore indirect solutions have been introduced on the market lately, see e.g., [1]. In this paper an indirect approach is presented where the tire radius is estimated simultaneously with the vehicle trajectory. This is done under the assumption that there is a relation between a reduction in tire radius and tire pressure.

The indirect approach presented in [1] is only based on the wheel speed sensors and it is shown how a tire pressure loss in one wheel leads to a relative radii error between the wheels. In later publications GPS measurements has also been included to improve the radius estimation and even make it possible to estimate the absolute radius of one tire. The effective tire radius is estimated using a simple leastsquares regression technique in [2]. A non-linear observer approach to estimate the tire radius is presented in [3], and a second order sliding mode observer is used to estimate the tire radius in [4]. A simultaneous maximum likelihood calibration and sensor pos estimation approach for mobile robots is presented in [5], where among other parameters the wheel radii are estimated.

In the present contribution the tire radius is assumed to be afflicted with Gaussian distributed noise, where both the mean and the covariance are unknown. The noise statistics

The authors would like to thank the Swedish Research Council under the Linnaeus Center CADICS for financial support.

The authors are with the Department of Electrical Engineering, Linköping University, Linköping, Sweden. \{emre, lundquist, fredrik\}@isy.liu.se are treated as unknown parameters and the trajectory of the vehicle is described by the state vector. The structure of this nonlinear problem with biased and unknown noise requires approximative algorithms for the state estimation. The particle filter provides one generic approach to nonlinear non-Gaussian filtering. Probably the most common way to handle a joint parameter and state estimation problem is augmenting the state vector with the unknown parameters and redefine the problem as a filtering problem, see e.g., [6], [7]. The approach has some major disadvantages as it requires artificial dynamics for the static parameters and it leads also to an increase in the state dimension which is not preferable for particle filters. This is particularly important to stress in automotive applications, where the computational cost must be kept low. In this work, an efficient Bayesian method is proposed for approximating the joint density of the unknown parameters and the state based on the particle filters and the marginalization concept, introduced in [8]. The statistics of the posterior distribution for the unknown noise parameters is propagated recursively and conditioned on the output of the particle filter.

The paper is outlined as follows. The problem is formulated together with the vehicle model and a description of the sensors in Section II. The estimation procedure and the filter approach is described in Section III. Results based on real data collected with a production type passenger car are presented in Section IV. The work is concluded in Section V.

\section{MODEL}

In this section we introduce the model used in estimating the pose trajectory of a four wheeled vehicle, where the angular velocities of the wheels and GPS positions are used as the inputs and the measurements, respectively. Our aim is to jointly estimate the state trajectory and the unknown tire radius errors given the measurements. The unknown wheel radii effect the state through the wheel speed sensor. The state vector is defined as the planar position and the heading angle of the vehicle,

$$
\mathbf{x}=\left[\begin{array}{lll}
\mathbf{x} & \mathrm{y} & \psi
\end{array}\right]^{\mathrm{T}}
$$

The discrete time model for the evolution of the state is given as,

$$
\begin{aligned}
\mathrm{x}_{k+1} & =\mathrm{x}_{k}+T \mathrm{v}_{k} \cos \psi_{k} \\
\mathrm{y}_{k+1} & =\mathrm{y}_{k}+T \mathrm{v}_{k} \sin \psi_{k} \\
\psi_{k+1} & =\psi_{k}+T \dot{\psi}_{k} .
\end{aligned}
$$




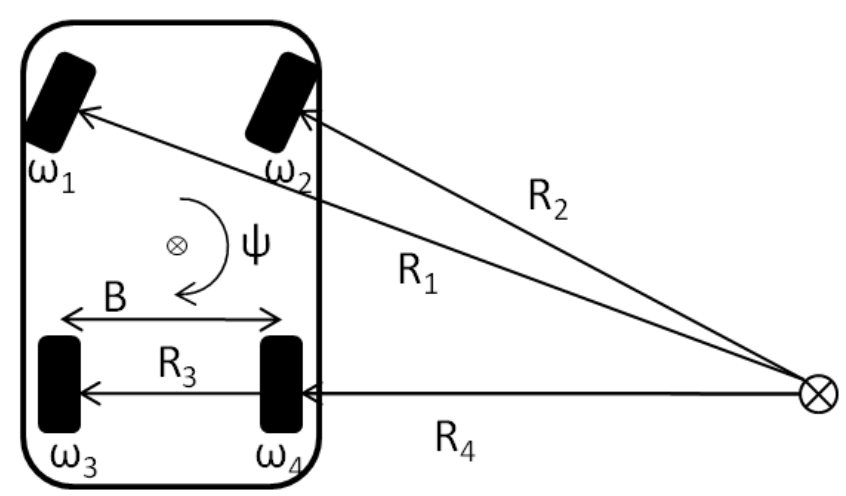

Fig. 1. The notation of the vehicle variables.

The available raw signals are the angular velocities of the wheels which can be measured by the ABS sensors. The angular velocities can be converted to virtual measurements of the absolute longitudinal velocity and yaw rate as described in [9], [10], assuming a front wheel driven vehicle with slipfree motion of the rear wheels,

$$
\begin{aligned}
\mathrm{v}^{\mathrm{virt}} & =\frac{w_{3} r_{3}+w_{4} r_{4}}{2} \\
\dot{\psi}^{\mathrm{virt}} & =\frac{w_{4} r_{4}-w_{3} r_{3}}{B} .
\end{aligned}
$$

See Figure 1 for the notation. In practice the actual tyre radii are unknown and need to be estimated on the run. The wheel radius errors are defined as the difference between the actual and the nominal values of the rear left and right wheel radii $\delta_{3} \triangleq r_{3}-r$ and $\delta_{4} \triangleq r_{4}-r$, respectively. Here, the nominal value of the wheel radius is denoted $r$ and equivalently

$$
\begin{aligned}
& r_{3}=r+\delta_{3} \\
& r_{4}=r+\delta_{4} .
\end{aligned}
$$

Substituting (4) in equations (3) results in

$$
\begin{aligned}
\mathrm{v}^{\text {virt }} & =\frac{\omega_{3} r_{3}+\omega_{4} r_{4}}{2}=\mathrm{v}+\frac{\omega_{3} \delta_{3}}{2}+\frac{\omega_{4} \delta_{4}}{2} \\
\dot{\psi}^{\text {virt }} & =\frac{\omega_{4} r_{4}-\omega_{3} r_{3}}{B}=\dot{\psi}+\frac{\omega_{4} \delta_{4}}{B}-\frac{\omega_{3} \delta_{3}}{B}
\end{aligned}
$$

The measurements defined as above have bias terms which are functions of $\delta_{3}$ and $\delta_{4}$. By using the virtual measurements as the inputs, the motion model (2) can be rewritten according to

$$
\begin{aligned}
& \mathrm{x}_{k+1}=\mathrm{x}_{k}+T\left(\mathrm{v}_{k}^{\text {virt }}-\frac{\omega_{3} \delta_{3}}{2}-\frac{\omega_{4} \delta_{4}}{2}\right) \cos \psi_{k} \\
& \mathrm{y}_{k+1}=\mathrm{y}_{k}+T\left(\mathrm{v}_{k}^{\text {virt }}-\frac{\omega_{3} \delta_{3}}{2}-\frac{\omega_{4} \delta_{4}}{2}\right) \sin \psi_{k} \\
& \psi_{k+1}=\psi_{k}+T\left(\dot{\psi}_{k}^{\text {virt }}-\frac{\omega_{4} \delta_{4}}{B}+\frac{\omega_{3} \delta_{3}}{B}\right) .
\end{aligned}
$$

The equations given above can be rewritten in the form of

$$
\begin{aligned}
& \mathbf{x}_{k}=f\left(\mathbf{x}_{k-1}, \mathbf{u}_{k}\right)+g\left(\mathbf{x}_{k-1}, \mathbf{u}_{k}\right) \mathbf{w}_{k}, \\
& \mathbf{y}_{k}=h\left(\mathbf{x}_{k}, \mathbf{u}_{k}\right)+\mathbf{e}_{k} .
\end{aligned}
$$

where where $\mathbf{u}_{k}$ is the virtual measurements used as the inputs and the noise term $\mathbf{w}_{k}$ is assumed to be Gaussian,

$$
\mathbf{w}_{k}=\mathcal{N}\left(\left(\begin{array}{c}
\delta_{3} \\
\delta_{4}
\end{array}\right),\left(\begin{array}{cc}
\Sigma_{3} & 0 \\
0 & \Sigma_{4}
\end{array}\right)\right) .
$$

The parameters aimed at estimating are the radii error bias and the covariances,

$$
\theta=\left\{\delta_{3}, \delta_{4}, \Sigma_{3}, \Sigma_{4}\right\}
$$

The sensor model (7b) is describing the relation with the GPS position and the state variables according to

$$
\left[\begin{array}{l}
\mathrm{x}_{k}^{\mathrm{GPS}} \\
\mathrm{y}_{k}^{\mathrm{GPS}}
\end{array}\right]=\left[\begin{array}{lll}
1 & 0 & 0 \\
0 & 1 & 0
\end{array}\right] \mathbf{x}_{k}+\mathbf{e}_{k} .
$$

where $\mathbf{e}_{k}$ is the measurement noise. In the following section we describe the estimation of the unknown bias terms and the covariances jointly with the state in a Bayesian framework.

\section{PARAmeter AND State Estimation}

In the Bayesian approach, we will utilize suitable prior distributions for the unknowns and compute the posterior joint density recursively. The inference will be done by using marginalized particle filters [11] where the unknown noise parameters are marginalized out in relevant steps. Our approach described here heavily relies on marginalization and conjugate priors concepts. Before introducing the details of the particle algorithm here we shortly repeat some preliminary information.

\section{A. Posterior Distribution for the Conjugate Prior}

For multivariate Normal data $z$ with unknown mean $\mu$ and covariance $\Sigma$, a Normal-inverse-Wishart distribution defines a conjugate prior $^{1}$. Let us denote it as $[\mu, \Sigma] \sim \operatorname{NiW}(\nu, V)$. The Normal-inverse-Wishart distribution defines a hierarchical Bayesian model given below:

$$
\begin{aligned}
z \mid \mu, \Sigma & \sim \mathcal{N}(\mu, \Sigma) \\
\mu \mid \Sigma & \sim \mathcal{N}(\hat{\mu}, \hat{\Sigma}) \\
\Sigma & \sim \operatorname{iW}(\nu-d, \Lambda)
\end{aligned}
$$

where $\mathrm{iW}($.$) denotes the Inverse Wishart distribution and$ $d$ denotes the dimension of measurement vector $z$. The parameters $\nu$ and $V$ represent the sufficient statistics and can be updated recursively with the new data. The relevant quantities are defined as,

$$
\begin{aligned}
& \hat{\mu} \triangleq V_{11}^{-1} V_{1 z} \\
& \hat{\Sigma} \triangleq V_{11}^{-1} \Sigma \\
& \Lambda \triangleq V_{z z}-V_{1 z} V_{11}^{-1} V_{z 1}, \\
& V \triangleq\left[\begin{array}{ll}
V_{z z} & V_{1 z} \\
V_{z 1} & V_{11}
\end{array}\right],
\end{aligned}
$$

where $V_{z z}$ is defined as the upper-left $d \times d$ sub-block of $V \in \mathbb{R}^{(d+1) \times(d+1)}$

\footnotetext{
${ }^{1} \mathrm{~A}$ family of prior distributions is conjugate to a particular likelihood function if the posterior distribution belongs to the same family as the prior.
} 
The joint density of $(\mu, \Sigma)$ is of the form

$$
\begin{aligned}
p(\mu, \Sigma) & =\operatorname{NiW}(\nu, V) \\
& =\frac{1}{c}|\Sigma|^{-\frac{\nu}{2}} \\
& \times \exp \left(-\frac{1}{2} \operatorname{tr}\left(\Sigma^{-1}\left[I_{d}, \mu\right] V\left[-I_{d}, \mu\right]^{T}\right)\right),
\end{aligned}
$$

where $c$ is the normalizing constant.

Via conjugacy, the posterior distribution is again a normalinverse-Wishart distribution with updated statistics. The update equations of the statistics are as follows,

$$
\begin{aligned}
& V_{k}=\lambda V_{k-1}+\left[\begin{array}{c}
z_{k} \\
1
\end{array}\right]\left[\begin{array}{ll}
z_{k}^{T} & 1
\end{array}\right], \\
& \nu_{k}=\lambda \nu_{k-1}+1 .
\end{aligned}
$$

where the scalar real number $0 \leq \lambda \leq 1$ is defined as the forgetting factor. The use of the forgetting factor corresponds to the application of an exponential window on the collected statistics with effective length $h=\frac{1}{1-\lambda}$. The statistics relies on roughly the measurements within the last $h$ frames/time instances. That allows the algorithm to adapt the changes in the noise statistics in time.

The recursive equations enable us to propagate the sufficient statistics easily in time. Furthermore, the predictive distribution for $z$ becomes a $t$-distribution for a NiW prior.

$$
p\left(z_{k} \mid z_{1: k-1}, \nu_{0}, V_{0}\right)=p\left(z_{k} \mid \nu_{k-1}, V_{k-1}\right)=\operatorname{St}_{\nu-d+1}(\hat{\mu}, \bar{\Lambda})
$$

where

$$
\begin{aligned}
& \hat{\mu}=V_{11}^{-1} V_{1 z} \\
& \bar{\Lambda} \triangleq \frac{\left(1+V_{11}\right)}{(\nu-d+1) V_{11}}\left(V_{z z}-V_{1 z} V_{11}^{-1} V_{z 1}\right)
\end{aligned}
$$

$\mathrm{St}_{v}(\mu, \Upsilon)$ is the multivariate student-t distribution with $v$ degrees of freedom, located at $\mu$ with scale parameter $\Upsilon$.

\section{B. Marginalization in nonlinear filtering}

Let us define NiW priors for the unknown process noise sequences of the system defined by (7a) and (7b). Our aim is to approximate the joint density $p\left(\mathbf{x}_{0: k}, \theta \mid \mathbf{y}_{0: k}\right)$ and allow marginalization if possible. The joint distribution of the states and the unknown parameters can be decomposed into the conditional distributions as follows.

$$
p\left(\mathbf{x}_{0: k}, \theta \mid \mathbf{y}_{0: k}\right)=p\left(\theta \mid \mathbf{x}_{0: k}, \mathbf{y}_{0: k}\right) p\left(\mathbf{x}_{0: k} \mid \mathbf{y}_{0: k}\right) .
$$

Suppose we approximate the distribution $p\left(\mathbf{x}_{0: k} \mid \mathbf{y}_{0: k}\right)$ by a set of $N$ particles and their weights as

$$
p\left(\mathbf{x}_{0: k} \mid \mathbf{y}_{0: k}\right) \simeq \sum_{i=1}^{N} \omega_{k}^{(i)} \delta_{\mathbf{x}_{0: k}^{(i)}}(.)
$$

For each particle we can compute analytical expressions for the posterior distribution of the unknown parameters of the process noise. The posterior follows the normal-inverseWishart distribution and the sufficient statistics are updated at each time step, for each particle, according to Equations (20a)-(20b) where we define the pseudo measurements $z_{k}$ as follows,

$$
z_{k} \triangleq g^{\dagger}\left(\mathbf{x}_{k-1}, u_{k}\right)\left(\mathbf{x}_{k}-f_{k}\left(\mathbf{x}_{k-1}, \mathbf{u}_{k}\right)\right)
$$

where $g^{\dagger}\left(\mathbf{x}_{k-1}, \mathbf{u}_{k}\right)$ is the pseudo-inverse of the matrix $g_{k}\left(\mathbf{x}_{k-1}, \mathbf{u}_{k}\right)$ in (7a). For a given state trajectory $\mathbf{x}_{0: k}$ and the measurements $\mathbf{y}_{0: k}$ the pseudo measurements can be computed directly. Using the sequential importance sampling scheme for propagating the particle approximation (23) leads to the standard weight update equation:

$$
\omega_{k}^{(i)}=\omega_{k-1}^{(i)} \frac{p\left(\mathbf{y}_{k} \mid \mathbf{x}_{k}^{(i)}\right) p\left(\mathbf{x}_{k}^{(i)} \mid \mathbf{x}_{0: k-1}^{(i)}, \mathbf{y}_{0: k-1}\right)}{q\left(\mathbf{x}_{k}^{(i)} \mid \mathbf{x}_{0: k-1}^{(i)}, \mathbf{y}_{0: k}\right)},
$$

where $q($.$) is the importance distribution from which we$ sample $\mathbf{x}_{k}^{(i)}$.

\section{State prediction}

In computing the state transition density $p\left(\mathbf{x}_{k} \mid \mathbf{x}_{0: k-1}, \mathbf{y}_{0: k-1}\right)$, one can utilize the posterior distribution of the unknown parameters that are computed for each particle. One important advantage of using conjugate priors reveals itself here as it is possible to integrate out unknown noise parameters as they follow normal-inverse-Wishart distribution.

$$
\begin{aligned}
& p\left(\mathbf{x}_{k} \mid \mathbf{x}_{0: k-1}, \mathbf{y}_{0: k-1}\right) \\
&=\int p\left(\mathbf{x}_{k} \mid \mathbf{x}_{k-1}, \theta\right) p\left(\theta \mid \mathbf{x}_{0: k-1}, \mathbf{y}_{0: k-1}\right) d \theta .
\end{aligned}
$$

For each particle $i$, the distribution can be written as:

$$
\begin{aligned}
p & \left(\mathbf{x}_{k} \mid \mathbf{x}_{0: k-1}^{(i)}, \mathbf{y}_{0: k-1}\right) \\
& =p_{z_{k}}\left(g^{\dagger}\left(\mathbf{x}_{k-1}, \mathbf{u}_{k}\right)\left(\mathbf{x}_{k}-f\left(\mathbf{x}_{k-1}^{(i)}, \mathbf{u}_{k}\right)\right) \mid \mathbf{x}_{0: k-1}^{(i)}, \mathbf{y}_{0: k-1}\right) \\
& =p_{z_{k}}\left(g^{\dagger}\left(\mathbf{x}_{k-1}, \mathbf{u}_{k}\right)\left(\mathbf{x}_{k}-f\left(\mathbf{x}_{k-1}^{(i)}, \mathbf{u}_{k}\right)\right) \mid z_{1: k-1}^{(i)}\right) \\
& =p_{z_{k}}\left(g^{\dagger}\left(\mathbf{x}_{k-1}, \mathbf{u}_{k}\right)\left(\mathbf{x}_{k}-f\left(\mathbf{x}_{k-1}^{(i)}, \mathbf{u}_{k}\right)\right) \mid \nu_{k-1}^{(i)}, V_{k-1}^{(i)}\right)
\end{aligned}
$$

The resulting predictive distribution is a multivariate Student$\mathrm{t}$ distribution given in (21).

At the sampling stage, in most of the cases it is not possible to sample from the optimal importance distribution. The state transition density $p\left(\mathbf{x}_{k} \mid \mathbf{x}_{0: k-1}, \mathbf{y}_{0: k-1}\right)$ can be used as the importance distribution. In that case the weight update equation (25) reduces to,

$$
\omega_{k}^{(i)}=\omega_{k-1}^{(i)} p\left(\mathbf{y}_{k} \mid \mathbf{x}_{k}^{(i)}\right) .
$$

Moreover computation of the pseudo inverse $g^{\dagger}\left(\mathbf{x}_{k-1}, \mathbf{u}_{k}\right)$ becomes no longer necessary. That is because when $q\left(\mathbf{x}_{k}^{(i)} \mid \mathbf{x}_{0: k-1}^{(i)}, \mathbf{y}_{0: k}\right)$ is chosen as $p\left(\mathbf{x}_{k} \mid \mathbf{x}_{0: k-1}, \mathbf{y}_{0: k-1}\right)$, one first samples from (21) in order to sample $x_{k}^{i}$. Then the samples from (21) can be used directly in the statistics update (20a)-(20b). The pseudo code of the simplified algorithm used in the simulations is given in Table I.

In the proposed method, each particle keeps its own estimate for the parameters of the unknown process noise 
TABLE I

PSEudo Code of THE Algorithm

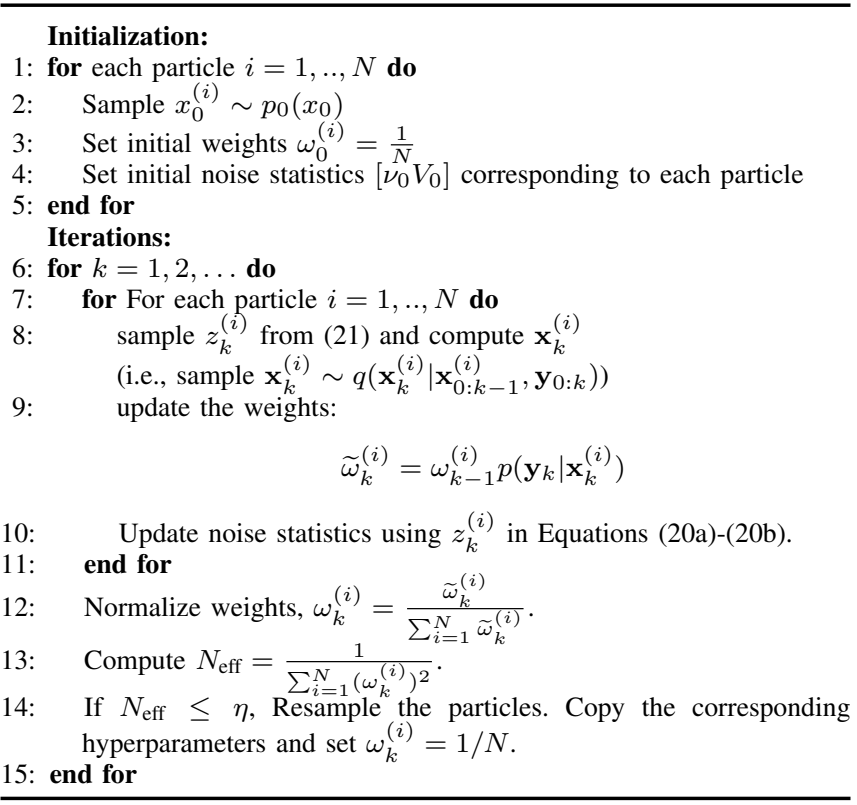

and the measurement noise. In the importance sampling step, the particles use their own posterior distribution of the unknown parameters. The weight update of the particles is made according to the measurement likelihood. The particles, keeping the unknown parameters which best explains/fits to the observed measurement sequence will survive in time.

\section{Posterior Distribution for the Noise Parameters}

The marginal posterior density of the unknown parameters can be computed by integrating out the states in the joint density.

$$
\begin{aligned}
p\left(\theta \mid \mathbf{y}_{1: k}\right) & =\int p\left(\theta \mid \mathbf{x}_{0: k}, \mathbf{y}_{1: k}\right) p\left(\mathbf{x}_{0: k} \mid \mathbf{y}_{1: k}\right) d \mathbf{x}_{0: k} \\
& \approx \sum_{i=1}^{N} \omega_{k}^{(i)} p\left(\theta \mid \mathbf{x}_{0: k}^{(i)}, \mathbf{y}_{1: k}\right) .
\end{aligned}
$$

Then the estimate of the unknown parameters could be computed according to a chosen criterion. As an example, according to the minimum mean square error (MMSE) criterion, the noise variance estimate at time $t$ could be computed as

$$
\widehat{\Sigma_{k}}=\sum_{i=1}^{N} \omega_{k}^{(i)} \frac{\Lambda_{k}^{(i)}}{v_{k}-d-1},
$$

where the weights are inherited from the particles.

\section{RESUlts}

Measurements were collected with a passenger car equipped with standard vehicle sensors, such as wheel speed sensors, and a GPS receiver, see Figure 2. The vehicle is further equipped with an additional and more accurate IMU, besides the standard IMU already mounted in the car, and an optical velocity sensor. These two additional sensors were

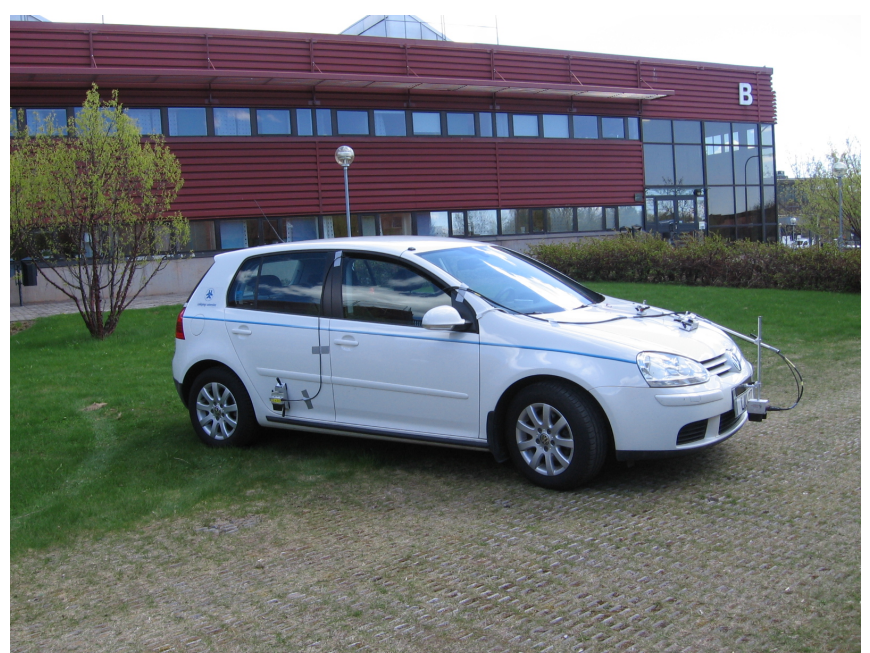

Fig. 2. The test vehicle of Linköping University is logging standard CAN data. The vehicle is in addition equipped with a GPS receiver, an IMU and an optical velocity sensor.

used to calibrate the setup, but were not further used to produce the results presented.

In regions where the car moves at low velocities, we utilize the steering wheel angle measurement as follows, in order to avoid quantization problems of the wheel cox

$$
\begin{aligned}
\mathrm{x}_{k+1} & =\mathrm{x}_{k}+T\left(\mathrm{v}_{k}^{\mathrm{virt}}-\frac{\omega_{3} \delta_{3}}{2}-\frac{\omega_{4} \delta_{4}}{2}\right) \cos \psi_{k} \\
\mathrm{y}_{k+1} & =\mathrm{y}_{k}+T\left(\mathrm{v}_{k}^{\mathrm{virt}}-\frac{\omega_{3} \delta_{3}}{2}-\frac{\omega_{4} \delta_{4}}{2}\right) \sin \psi_{k} \\
\psi_{k+1} & = \begin{cases}\psi_{k}+T\left(\dot{\psi}_{k}^{\mathrm{virt}}-\frac{\omega_{4} \delta_{4}}{B}+\frac{\omega_{3} \delta_{3}}{B}\right) & \text { if } \mathrm{v}>\gamma \\
\psi_{k}+T \delta_{F}\left(\mathrm{v}_{k}^{\mathrm{virt}}-\frac{\omega_{3} \delta_{3}}{2}-\frac{\omega_{4} \delta_{4}}{2}\right) / l_{b} & \text { if } \mathrm{v}<\gamma\end{cases}
\end{aligned}
$$

The GPS measurements of the $12 \mathrm{~km}$ test round is shown as a black solid line in Figure 3. The round took about $18 \mathrm{~min}$ to drive and it starts and ends in urban area of Linköping, in the upper right corner in Figure 3. The test vehicle is driving clockwise, first on a small rural road, and then on the left side of the figure entering a straight national highway, before driving back to urban area on the top of the figure. The gray dashed line shows the estimated trajectory of the vehicle.

For the first round the pressure of the rear wheel tires was adjusted to be equal 2.8 bar on both tires. For the second round the pressure of the rear left tire was released to 1.5 bar. The estimated parameters, i.e., the mean and the covariance for the left and the right wheel are shown in the Figures 4 and 5, respectively. The black solid line shows the estimated mean and covariance for the balanced pressure case, whereas the gray line shows the estimated parameters for the second round where the left tire radius was released by almost $50 \%$. Note that the pressure loss of the left wheel reduces the estimated tire radius error $\delta_{3}$ by approximately $1.5 \mathrm{~mm}$, see Figure 4. However, the estimated tire radius error of the right wheel, which has the same tire pressure for both experiment, keeps its value, see Figure 5 


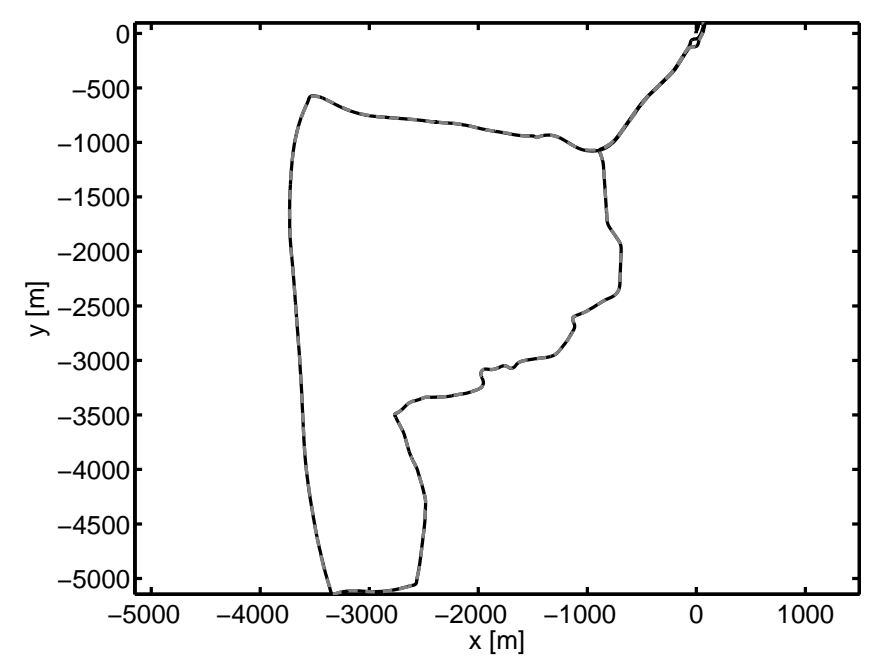

Fig. 3. The black line is GPS position measurements and the gray line is the estimated driven trajectory. The experiment starts and ends at a roundabout in the upper right corner.
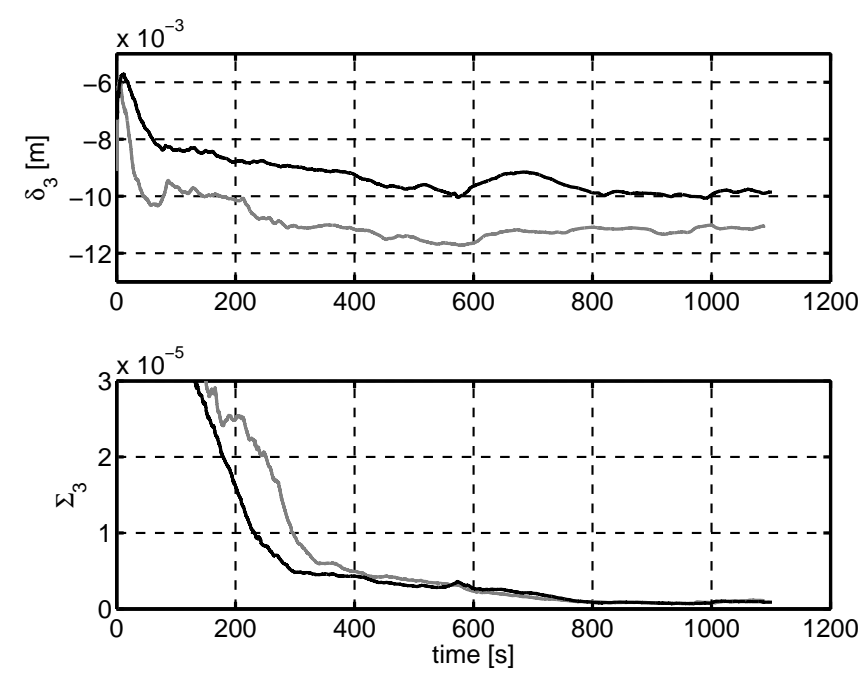

Fig. 4. Tire radius error of the left rear wheel. The upper plot shows the mean value $\delta_{3}$ and the lower plot the covariance estimate $\Sigma_{3}$. The black solid line is the estimated values from the first experiment, with balanced wheel pressure, and the gray line shows the estimate from the second experiment where the pressure of the left wheel is reduced by $50 \%$. The tire pressure loss lead to a radius reduction of about $1.5 \mathrm{~mm}$.

The results are even more apparent in Figure 6, where the difference between the left and the right tire radius error, i.e., $\delta_{3}-\delta_{4}$, is shown. In the first experiment with balanced wheel pressure the difference is around zero, whereas in the second experiment the tire radius difference is around $1.5 \mathrm{~mm}$. Besides the the situations where the vehicle is moving slowly and cornering, i.e., the junctions at $500-600 \mathrm{~s}$ and the roundabout at $1000-1100 \mathrm{~s}$ it is possible to estimate the relative tire radius difference with sub-millimeter accuracy.

\section{CONCLUSION}

In this study, we address the problem of joint estimation of unknown tire radii and the trajectory of a four wheeled vehicle based on GPS and wheel angular velocity measure-
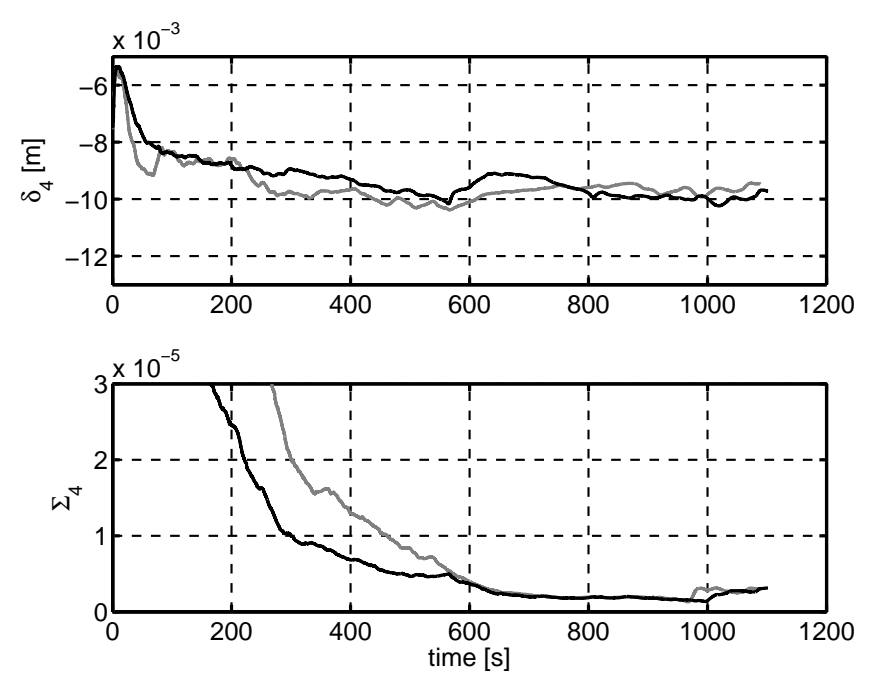

Fig. 5. Tire radius error of the right rear wheel. The upper plot shows the mean value $\delta_{4}$ and the lower plot the covariance estimate $\Sigma_{4}$. The black solid line is the estimated values from the first experiment, with balanced wheel pressure, and the gray line shows the estimate from the second experiment where the pressure of the right wheel is unchanged.

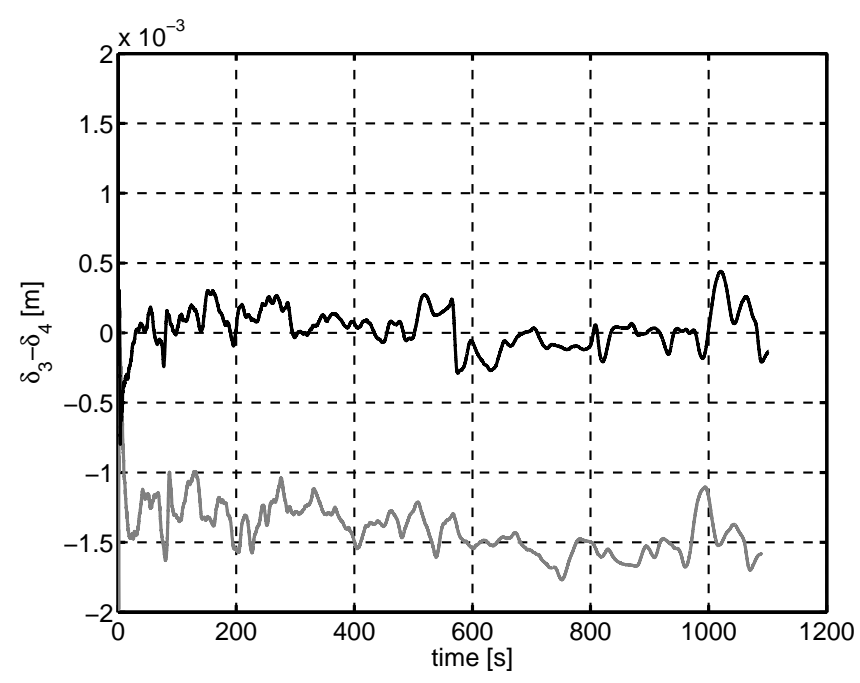

Fig. 6. Tire radius error difference between the left and the right rear wheels. The black solid line is the estimated values from the first experiment, with balanced wheel pressure, and the gray line shows the estimate from the second experiment where the pressure of the left wheel is reduced by $50 \%$. The tire pressure loss lead to a radius reduction of about $1.5 \mathrm{~mm}$

ments. The problem is defined in Bayesian framework and an efficient method that utilizes marginalized particle filters is proposed in order to accomplish the difficult task of joint parameter and state estimation. The algorithm is tested on real data experiments. The results show that it is possible to estimate relative tire radius difference within sub-millimeter accuracy.

\section{ACKNOWLEDGMENTS}

The Authors would like to thank Kristoffer Lundahl, at the vehicular systems division at Linköpings University, for helping us with the data collection. 


\section{REFERENCES}

[1] N. Persson, S. Ahlqvist, U. Forssell, and F. Gustafsson, "Low tire pressure warning system using sensor fusion," in Proceedings of the Automotive and Transportation Technology Congress, ser. SAE paper 2001-01-3337, Barcelona, Spain, Oct. 2001.

[2] S. Miller, B. Youngberg, A. Millie, P. Schweizer, and J. Gerdes, "Calculating longitudinal wheel slip and tire parameters using gps velocity," in Procedings of the IEEE American Control Conference, vol. 3, Jun. 2001, pp. 1800-1805.

[3] C. Carlson and J. Gerdes, "Consistent nonlinear estimation of longitudinal tire stiffness and effective radius," IEEE Transactions on Control Systems Technology, vol. 13, no. 6, pp. 1010-1020, Nov. 2005.

[4] N. M'sirdi, A. Rabhi, L. Fridman, J. Davila, and Y. Delanne, "Second order sliding mode observer for estimation of velocities, wheel sleep, radius and stiffness," in Procedings of the IEEE American Control Conference, Jun. 2006, pp. 3316-3321.

[5] A. Censi, L. Marchionni, and G. Oriolo, "Simultaneous maximumlikelihood calibration of odometry and sensor parameters," in Proceedings of the IEEE International Conference on Robotics and Automation, Pasadena, Canada, May 2008, pp. 2098-2103.

[6] J. Liu and M. West, "Combined parameter and state estimation in simulation-based filtering," in Sequential Monte Carlo Methods in Practice, A. Doucet, N. D. Freitas, and N. Gordon, Eds. Springer, 2001.

[7] S. Julier and H. Durrant-Whyte, "Process models for the high-speed navigation of road vehicles," in Proceedings of the IEEE International Conference on Robotics and Automation, vol. 1, May 1995, pp. 101105.

[8] S. Saha, E. Özkan, F. Gustafsson, and V. Smidl, "Marginalized particle filters for bayesian estimation of gaussian noise," in Proceedings of the International Conference on Information Fusion, Edinburgh, Scotland, Jul. 2010.

[9] F. Gustafsson, S. Ahlqvist, U. Forssell, and N. Persson, "Sensor fusion for accurate computation of yaw rate and absolute velocity," in Proceedings of the SAE World Congress, ser. SAE paper 2001-011064, Detroit, MI, USA, Apr. 2001.

[10] F. Gustafsson, Statistical Sensor Fusion. Lund, Sweden: Studentlitteratur, 2010.

[11] T. B. Schön, F. Gustafsson, and P.-J. Nordlund, "Marginalized particle filters for mixed linear/nonlinear state-space models," IEEE Transactions on Signal Processing, vol. 53, no. 7, pp. 2279-2289, Jul. 2005. 